Instituto Internacional de Investigación y Desarrollo Tecnológico Educativo INDTEC, C.A.

DOI: https://doi.org/10.29394/Scientific.issn.2542-2987.2019.4.13.3.60-77

OAI-PMH: http://www.indteca.com/ojs/index.php/Revista Scientific/oai

Artículo Original / Original Article

\title{
El Posicionamiento en Motores de Búsqueda en Empresas Hoteleras del Cantón Sucre: Ecuador 2018
}

\author{
Autores: Frank Ángel Lemoine Quintero \\ Universidad Laica "Eloy Alfaro" de Manabí, Extensión Bahía de Caráquez, ULEAM \\ fangel64@gmail.com \\ Bahía de Caráquez, Ecuador \\ https://orcid.org/0000-0001-8885-8498 \\ Cristian Eduardo Medranda Vera \\ Universidad Laica "Eloy Alfaro" de Manabí, Extensión Bahía de Caráquez, ULEAM \\ cristian.medranda@hotmail.com \\ Bahía de Caráquez, Ecuador \\ https://orcid.org/0000-0001-9073-091X \\ Gema Viviana Carvajal Zambrano \\ Universidad Laica "Eloy Alfaro" de Manabí. Extensión Bahía de Caráquez, ULEAM \\ vivicarvajalzambrano@gmail.com \\ Bahía de Caráquez, Ecuador \\ https://orcid.org/0000-0001-8451-9683 \\ César Arturo Carbache Mora \\ Universidad Laica "Eloy Alfaro" de Manabí. Extensión Bahía de Caráquez, ULEAM \\ cesarcarbache@gmail.com \\ Bahía de Caráquez, Ecuador \\ https://orcid.org/0000-0001-9373-2873
}

\section{Resumen}

La investigación persiguió el objetivo de evaluar la presencia a nivel de plataforma web las empresas de servicios hoteleros existentes en la zona objeto estudio. Se fundamenta primeramente en el análisis del catastro y levantamiento realizado por la Extensión Bahía de Caráquez. El estudio se estructura en tres fases, en la primera se aplicó el método deductivo que a su vez se procedió evaluar la presencia web a partir de las categorías de las empresas hoteleras, determinando que el $77 \%$ de las empresas no cuentan con representatividad a este nivel. En la segunda fase se realizó la auditoría SEO para medir el nivel de optimización a través de 23 métricas, donde en el cuadrante superado estuvo representado por un total de $50 \%$ de aciertos, obteniendo un $22 \%$ de aciertos en el cuadrante de error y en el cuadrante de advertencia un total de $28 \%$ aciertos por lo que estos pueden ser superados a través de especialista en la temática que aporten a mejorar el posicionamiento web. La tercera fase se verificó el nivel de tráfico de clientes identificando el mejor evaluado fue la Casa del Sol representado por un $100 \%$ de redes sociales y Chirije con un $89,34 \%$ de tráfico orgánico, solo el $10,66 \%$ alcanzo un nivel de representatividad en redes sociales, las demás empresas hoteleras se mantienen con un $100 \%$ en el tráfico orgánico.

Palabras clave: tráfico; servicios; auditoría de gestión.

Fecha de Recepción: 18-03-2019
Fecha de Aceptación: 29-06-2019
Fecha de Publicación: 05-08-2019 


\title{
Positioning in Search Engines in Hotel Companies of Cantón Sucre: Ecuador 2018
}

\begin{abstract}
The research pursued the objective of evaluating the presence at the web platform level of hotel services companies in the area under study. It is based primarily on the analysis of the cadastre and survey carried out by the Bahía de Caráquez Extension. The study is structured in three phases, in the first one the deductive method was applied, which in turn proceeded to evaluate the web presence from the categories of the hotel companies, determining that $77 \%$ of the companies do not have representativeness to this level. In the second phase, the SEO audit was performed to measure the level of optimization through 23 metrics, where in the quadrant exceeded was represented by a total of $50 \%$ correct answers, obtaining $22 \%$ of correct answers in the error quadrant and in the warning quadrant a total of $28 \%$ correct so these can be overcome through specialist in the subject that contribute to improve the web positioning. The third phase was verified the level of customer traffic identifying the best evaluated was the Casa del Sol represented by $100 \%$ social networks and Chirije with $89.34 \%$ organic traffic, only $10.66 \%$ reached a level of representativeness in social networks, the other hotel companies remain $100 \%$ in organic traffic.
\end{abstract}

Keywords: traffic; services; management audit.

Date Received: 18-03-2019
Date Acceptance:

29-06-2019
Date Publication:

05-08-2019 


\section{Introducción}

El marketing digital ha tomado notabilidad a nivel empresarial por lo que hoy en día tiene mucha relevancia y representatividad en las empresas hoteleras, es importante reconocer que estos sitios donde se encuentran ubicada las empresas conforman visibilidad de un sistema con la intensión de ser diseñado para producir una interacción fructífera de similitud entre dicho sistema y usuarios.

Según Andrade (2016): en su estudio realizado sobre estrategia de marketing digital fundamenta desde la teoría de diferentes autores que en principio aborda desde la capacidad innovadora hasta el rol de las Tecnologías de la Información y las Comunicaciones (TIC's) se han generalizado en el sector turístico, formando una herramienta elemental que aporta a promocionar desde las aplicaciones móviles, portales web y website. (pág. 59).

Al respecto, Cárdenas (2015): realiza un estudio referente al marketing digital como una estrategia generadora de la innovación empresarial Bic Data Center, donde fundamenta que la estrategia considerada en su estudio alcanzó resultados relevantes de difusión utilizando como herramienta el internet por sus cualidades de recepción y transmisión de información de forma continua (pág. 9).

Las redes sociales constituyen el servicio que ha revolucionado las comunidades virtuales, desde su perspectiva y contingencias de crecimiento, donde se debe de incursionar en actividades que permitan desarrollar estrategias de posicionamiento que lo direccionen a un mercado cada vez más competitivo en busca de nuevas oportunidades a partir del Marketing MIX.

De acuerdo con Miranda, Cruz, Valle y Flores (2015): realizan una reflexión referente al uso del comercio electrónico en el sector turístico como una alternativa que optimiza las acciones tradicionales, enfatiza que el turismo electrónico se ha direccionado a obtener resultados de las demandas en las actividades del ocio y la tecnología con la finalidad de introducir nuevos 
modelos de gestión sustentados en la satisfacción al cliente o consumidores (pág. 12).

Es importante reconocer que el posicionamiento web no es más que la consecuencia de la recuperación de información en base de datos ubicados en grandes Motores de Búsqueda de Internet debido al uso de algoritmos de búsqueda en el software que permite realizar estudios referentes a empresas u organizaciones a nivel global. Es considerado también en el ámbito por permitir emplear disímiles técnicas que expanden a alcanzar que los buscadores instalen una posición y categoría alta (primera posición) definiendo resultados dentro de su página según los términos y frases clave de indagación que sean ejecutados.

Es importante referenciar la siguiente definición de Marcos y Codina (2005):

POSICIONAR es colocar alguna cosa en su lugar óptimo. En el ámbito de la world wide web, posicionar un sitio significa optimizarlo para que aparezca en las primeras posiciones de las páginas de resultados de los motores de búsqueda. Así mismo, podemos definir posicionamiento web como el conjunto de procedimientos y técnicas que tienen como finalidad dotar a un sitio o a una página web de la máxima visibilidad en Internet (pág. 84).

Diferentes criterios de autores sobre el posicionamiento SEO, desde su concepción natural u orgánico lo cual figura similar y consiste en utilizar un sin número de estrategias, libre de pago en publicidad, con el propósito de que sitios web conquisten lugares privilegiados como resultados de los buscadores, esto permitirá que el servicio, paginas o empresas desde la web ocupen un lugar en el que tendrá un número de clientes o visualizadores.

El Ministerio del Turismo del Ecuador (MINTUR, 2018): creó la Subsecretaría de Inversión y Fomento Turístico en el año 2014, con la finalidad de impulsar estrategias enfocada a posicionar al país en un mercado 
internacional, gestionando al destino como líder de inversiones turísticas; delineado políticas y estrategias que están conducentes a adquirir la atracción a nivel nacional e internacional (pág. 4).

En los últimos años, la actividad de servicios hoteleros del país ha representado un rublo significante según fuentes estadísticas del portal de servicios del MINTUR representa un crecimiento en el país de un 14\% del año 2018 respecto al año anterior. Este creciente movimiento turístico, así como un incremento en cadenas internacionales e inversionistas nacionales le han apostado a Ecuador como un destino privilegiado.

El cantón Sucre se encuentra ubicado al Noreste de la provincia de Manabí, tiene una población de 57.159 habitantes, con tres parroquias y su cabecera cantonal que es la ciudad de Bahía de Caráquez. Existen 53 empresas hoteleras las cuales tienen la denominación de: Hoteles, Hostales, Cabañas, Moteles y Pensiones.

Los servicios web se han convertido en fuente de posicionamiento altamente competitivo en los mercados tanto a nivel nacional como internacional haciendo evidente que empresas de servicios hoteleros en el cantón Sucre donde solo 12 de estas empresas hoteleras constan con páginas web que representa el $23 \%$ y un $77 \%$ prescinde con presencia web, pero sí un $30 \%$ de empresas hoteleras utilizan website.

La ausencia de página web en las empresas que brindan servicios hoteleros no ha permitido que estos servicios no opten por un posicionamiento web tanto en el mercado hotelero como turístico a nivel nacional e internacional, representado por una disminución de un $20 \%$ del nivel de ocupación.

La investigación esta direccionado a evaluar el posicionamiento de los motores de búsqueda de las empresas que brindan servicios hoteleros en el cantón Sucre, estructurada esta investigación en tres fases la primera con la finalidad de verificar la presencia online de las empresas que brindan servicios 
turísticos, la segunda fase es evaluar la presencia web de empresas hoteleras a través de plataformas que miden parámetros SEO estandarizados a través de la auditoria conformada por tres cuadrantes y la tercera fase será con la finalidad de medir posicionamiento para jerarquizar el posicionamiento de las 3 primeras empresas de servicio hotelero.

No existe una fórmula infalible para lograr que nuestra página web se ubique entre las primeras posiciones de la lista de resultados de búsqueda, pero sí podemos conseguir mejorar las posiciones si tenemos en cuenta una serie de aspectos sobre todo a nivel de diseño y contenido de la página web, para lograr mejores resultados. Por lo que este estudio se fundamenta en evaluar el posicionamiento web del sector hotelero para verificar si cumplen con los parámetros web para que tengan un lugar competitivo en el mercado internacional con la finalidad de desarrollar estrategias a nivel de la web.

\section{Metodología}

La investigación se estructura en tres fases con la finalidad de buscar un procedimiento que permita evaluar a través de parámetros definidos los hoteles de la zona objeto estudio. La primera fase de diagnóstico concebida por un levantamiento de infraestructura, la segunda fase evaluativa permitirá categorizar el número de empresas hoteleras con presencia web en la zona y la tercera fase realizará el análisis de la auditoria de posicionamiento SEO On Page para un mayor análisis de las empresas y definir las métricas del nivel de optimización web.

Se utilizara el método deductivo a partir del análisis de las empresas hoteleras existentes en la localidad partiendo de estadísticos de levantamiento donde el $20 \%$ corresponde a la primera categoría, el $40 \%$ que representa la segunda categoría y finalmente el $40 \%$ restante que representan la tercer categoría, de estos resultados se verificara el posicionamiento a nivel de página web de los hoteles, hostales, cabañas y moteles cumplen con las 
métricas SEO para determinar la presencia de los mismos.

\section{Desarrollo}

Estadísticas demuestran que a partir del terremoto suscitado en abril del año 2016, el Cantón Sucre contaba con un mayor número de establecimientos hoteleros, un $65 \%$ fueron afectados, donde el $30 \%$ de estos establecimientos hoteleros eran pensiones seguidas por los hostales con un $21 \%$, los hoteles y hostales residenciales con un $13 \%$ cada uno y las hosterías y cabañas con un $11 \%$ (tomando en cuenta que esta clasificación por categorías: pensiones, hoteles, hosterías, hostales, hostales residenciales, cabañas, es de acuerdo al Reglamento General de Actividades Turísticas del año 2008).

Es significativo reflejar que estudios realizados de levantamiento estadísticos demuestran que las pensiones representan una mayor representatividad a nivel de empresas de servicios de hospedaje con un $21.7 \%$ y con un nivel de ocupación de un $20.8 \%$, seguido de los hoteles, hostales y hostales residenciales con un $17.4 \%$ aunque su nivel de ocupación varia de $19.5 \%$ a un $22.3 \%$ en los servicios hoteleros. Las hosterías representaron en $13 \%$ al igual que las Cabañas debido a que el servicio turístico en la zona es considerado como un turismo rural, comunitario y de aventura, tal como se representa en la tabla 1.

Tabla 1. Estudio a nivel de empresas hoteleras.

\begin{tabular}{|l|c|c|c|c|}
\hline CLASIFICACIÓN & CANTIDAD & PORCENTAJE & $\begin{array}{c}\text { NIVEL DE } \\
\text { OCUPACIÓN }\end{array}$ & $\begin{array}{c}\% \text { DE } \\
\text { OCUPACIÓN }\end{array}$ \\
\hline HOTEL & 4 & $17.4 \%$ & 194 & $19.5 \%$ \\
\hline HOSTAL & 4 & $17.4 \%$ & 207 & $20.8 \%$ \\
\hline $\begin{array}{l}\text { HOSTAL } \\
\text { RESIDENCIAL }\end{array}$ & 4 & $17.4 \%$ & 222 & $22.3 \%$ \\
\hline HOSTERÍA & 3 & $13.0 \%$ & 95 & $9.5 \%$ \\
\hline
\end{tabular}




\begin{tabular}{|l|c|c|c|c|} 
PENSIÓN & 5 & $21.7 \%$ & 207 & $20.8 \%$ \\
\hline CABAÑA & 3 & $13.0 \%$ & 70 & $7.0 \%$ \\
\hline TOTAL & 23 & $100 \%$ & 995 & $100 \%$ \\
\hline
\end{tabular}

Fuente: Los Autores (2017).

Una vez obtenido los resultados de las empresas hoteleras existente en el cantón se procedió a evaluar el posicionamiento web a través de la plataforma virtual con la finalidad de visualizar las empresas de servicios hoteleros que tienen presencia web, esto se realizó a nivel de categoría de cada una de las empresas que brindan el servicio turístico, la información tomada del catastro del Gobierno Autónomo Descentralizado del Cantón Sucre (GADS) y del levantamiento que se realizó por parte Ministerio de Desarrollo Urbano y de Vivienda del Ecuador (MIDUVI) en coordinación con estudiantes investigadores del campus de Bahía de Caráquez de la Universidad Laica "Eloy Alfaro" de Manabí (ULEAM), después del evento telúrico del 16A.

Para evaluar el posicionamiento web en las empresas hoteleras se realizará a través de un análisis estadístico donde estará representado según las categorías designada por el Ministerio de Turismo del Ecuador para empresas hoteleras y su representatividad en la página web en dependencia de la auto gestión del negocio, tal como se muestra en la tabla 2.

Tabla 2. Hoteles con presencia web.

\begin{tabular}{|l|c|c|c|c|c|c|}
\hline Categoría & $\mathbf{N}^{\circ}$ Empresas & Representatividad \% & Si & $\%$ & No & $\%$ \\
\hline Primera & 6 & $11 \%$ & 6 & $100 \%$ & 0 & $0 \%$ \\
\hline Segunda & 13 & $25 \%$ & 2 & $15 \%$ & 11 & $85 \%$ \\
\hline Tercera & 34 & $64 \%$ & 4 & $12 \%$ & 30 & $88 \%$ \\
\hline Total & 53 & $100 \%$ & 12 & ------ & 41 & ------ \\
\hline
\end{tabular}

Fuente: Los autores (2018).

En el análisis que se hizo referente a la primera categoría, existen 6 empresas corresponden al servicio hotelero y que a su vez representa un $11 \%$ de representatividad que a su vez estas representaron un $100 \%$ de presencia 
web. La segunda categoría representa simbolizo el $25 \%$ de empresas hoteleras, pero solo de estas el 15\% mostraron presencia web y un $85 \%$ no mostró presencia o páginas web. Es distintivo como en la tercera categoría las empresas hoteleras están representada por un 64\%, donde de estas solo el $12 \%$ posee presencia web y un $88 \%$ no consta con presencia. De un total de 53 empresas hoteleras resulto que existen 41 que no tienen presencia web que representa $77 \%$ de la totalidad sin el servicio, siendo representativo para posicionar el turismo a escala nacional e internacional.

\subsection{Análisis de auditoria de posicionamiento SEO On Page.}

Con la finalidad de mejorar en el posicionamiento web de las empresas hoteleras en el cantón se realiza la auditoria a partir de las siguientes métricas tales como: Url, canonical, titulo, meta, descripción, palabras clave meta, encabezados, imágenes, relación text-html, marcos, flash, microformatos, schema.org, open graph, tarjeta de twitter, compatibilidad con dispositivos móviles, accelerated mobile pages, meta-viewport, cumplimiento del sitio, robots.txt, sistemas $\mathrm{xml}$, idioma, tipo de formato, codificación, google analytics e icono de página, entre los más representativos.

Las métricas enunciadas anteriormente son el resultado del nivel de optimización de la página, que se evaluará a través de tres parámetros el superado, el de error y el de advertencia, según la representatividad del número de métrica en cada uno de estos cuadrantes definirá cuan bien, regular o mal está el posicionamiento de la página. Esta evaluación se realizará a nivel de hoteles, hostales, moteles, cabañas y pensión en dependencia del estudio realizado anteriormente.

A continuación, mostramos la auditoria SEO, la cual permitirá observar rápida y fácilmente el estado de optimización de la web, la cual es realizada en las empresas de servicios hoteleros del cantón las cuales se representan en la tabla 3. 
Tabla 3. Resultados de la auditoria SEO On Page (Hoteles y Hostales)

\begin{tabular}{|c|c|c|c|}
\hline Hoteles & Superado & Error 黹 & Advertencia $\theta$ \\
\hline La Herradura & 11 & 6 & 6 \\
\hline La Piedra & 15 & 2 & 6 \\
\hline \multicolumn{4}{|l|}{ Hostales } \\
\hline Casa Ceibo & 16 & 3 & 4 \\
\hline Delfines Beach & 12 & 3 & 8 \\
\hline San Jacinto & 8 & 6 & 9 \\
\hline Pensiones & Superado $\mathrm{W}$ & Error $\underline{\Delta}$ & Advertencia $\theta$ \\
\hline Coco Bongo & 10 & 6 & 7 \\
\hline La Casa del Sol & 17 & 3 & 3 \\
\hline Chelita & 10 & 7 & 6 \\
\hline Marco`s & 12 & 3 & 8 \\
\hline Palma Azul & 8 & 9 & 6 \\
\hline Cabañas & Superado $\mathbb{W}$ & Error $\stackrel{\Delta}{M}$ & Advertencia $\theta$ \\
\hline Chirije & 10 & 6 & 7 \\
\hline \multicolumn{4}{|l|}{ Hostelería } \\
\hline Costa del Sol Marco's & 10 & 7 & 6 \\
\hline
\end{tabular}

Fuente: Los Autores (2018).

En categoría de hoteles el hotel La Piedra posee mejor SEO, debido a que solo tiene 2 errores y seis advertencias; con referencia al hotel La Herradura que presenta 6 errores y 6 advertencias. Dentro de los errores se encuentran el duplicado de etiquetas de título, contenido duplicado, enlaces internos rotos, enlaces externos rotos, imágenes internas rotas y meta descripciones duplicada. En las advertencias las más representativas resultaron elemento del título largo y los atributos ALT proporciona textos alternativos que faltan por mostrar, determinando una presencia web que no cumple con las métricas pertinentes.

En categoría de hostal, la Casa Ceibo tiene el mejor posicionamiento con relación al hostal Delfines Beach y hostal San Jacinto según los parámetros medidos, los cuales tienen 3 errores y su diferencia está en las advertencias que son las mismas que las anteriores. 
En categoría a nivel de pensiones, La Casa del Sol, se encuentra en el primer lugar en SEO, con referencia a Marco's, Coco bongo, Chelita y Palma Azul. En categoría de Cabañas, La Cabaña Chirije, tiene el mismo nivel en la optimización del motor de búsqueda SEO, que la hostelería Costa del Sol Marco's, pero si observamos el índice de errores, Chirije tiene un error menos.

Por lo que según los parámetros medidos existen un total de 139 aciertos en el cumplimiento de las métricas en el cuadrante superado, por lo que en el cuadrante de error fue un total de 61 aciertos y en el parámetro de advertencia un total de 76 aciertos, denotando que el posicionamiento web de estas empresas hoteleras no es buena, por lo que se reconoce que la ausencia de visitas e impacto de la web de cada hotel carece de calidad de diseño y de posicionamiento que hace que la afluencia de turista no sea representativa en los mismos.

\subsection{Análisis de niveles de tráficos para definir posicionamiento web.}

Con la finalidad de profundizar en la temática se realizó a través de descripción de los canales de marketing la evaluación de 7 indicadores que son pertinentes para un buen posicionamiento web, tabla 4 .

Tabla 4. Descripción de los canales de Marketing.

\begin{tabular}{|l|c|c|c|c|c|c|c|}
\hline \multicolumn{1}{|c|}{ Hoteles } & $\begin{array}{c}\text { Tráfico } \\
\text { directo }\end{array}$ & $\begin{array}{c}\text { Enlaces } \\
\text { internos }\end{array}$ & $\begin{array}{c}\text { Enlaces } \\
\text { externos }\end{array}$ & $\begin{array}{c}\text { Tráfico } \\
\text { orgánico }\end{array}$ & $\begin{array}{c}\text { Redes } \\
\text { sociales }\end{array}$ & mail & $\begin{array}{c}\text { Display: } \\
\text { Banner }\end{array}$ \\
\hline La herradura & $0.00 \%$ & 46 & 5 & $0.00 \%$ & $0.00 \%$ & $0.00 \%$ & $0.00 \%$ \\
\hline La piedra & $0.00 \%$ & 25 & 5 & $100 \%$ & $0.00 \%$ & $0.00 \%$ & $0.00 \%$ \\
\hline \multicolumn{7}{|c|}{ Hostales } \\
\hline Casa Ceibo & $0.00 \%$ & 21 & 4 & $100 \%$ & $0.00 \%$ & $0.00 \%$ & $0.00 \%$ \\
\hline $\begin{array}{l}\text { Delfines } \\
\text { Beach }\end{array}$ & $0.00 \%$ & 9 & 2 & $0.00 \%$ & $0.00 \%$ & $0.00 \%$ & $0.00 \%$ \\
\hline San Jacinto & $0.00 \%$ & 20 & 7 & $100 \%$ & $0.00 \%$ & $0.00 \%$ & $0.00 \%$ \\
\hline
\end{tabular}




\begin{tabular}{|l|c|c|c|c|c|c|c|}
\hline \multicolumn{7}{|c|}{ Pensiones } \\
\hline Coco Bongo & $0.00 \%$ & 9 & 4 & $100 \%$ & $0.00 \%$ & $0.00 \%$ & $0.00 \%$ \\
\hline $\begin{array}{l}\text { La Casa del } \\
\text { Sol }\end{array}$ & $0.00 \%$ & 45 & 5 & $0.00 \%$ & $100 \%$ & $0.00 \%$ & $0.00 \%$ \\
\hline Chelita & $0.00 \%$ & 25 & 20 & $0.00 \%$ & $0.00 \%$ & $0.00 \%$ & $0.00 \%$ \\
\hline Marco'S & $0.00 \%$ & 14 & 36 & $100 \%$ & $0.00 \%$ & $0.00 \%$ & $0.00 \%$ \\
\hline Palma Azul & $50.57 \%$ & 13 & 3 & $19.24 \%$ & $30.19 \%$ & $0.00 \%$ & $0.00 \%$ \\
\hline \multicolumn{7}{|c|}{ Cabañas } \\
\hline Chirije & $0.00 \%$ & 43 & 2 & $89.34 \%$ & $10,66 \%$ & $0.00 \%$ & $0.00 \%$ \\
\hline \multicolumn{7}{|c|}{ Hostelería } \\
Marco'S
\end{tabular}

Fuente: Los Autores (2018).

Esta evaluación fue realizada en tres meses consecutivos (diciembre del año 2016, enero y febrero del año 2017), con la finalidad de que los resultados sean los más actualizados posible a nivel de empresa para definir donde existen mayores niveles de error y de advertencia. Por lo que los resultaros fueron los siguientes:

1. La fuente de tráfico de clientes para la Casa del Sol es en un $100 \%$ de redes sociales; Palma Azul tiene un 50, 57\% de tráfico directo, 30,19\% de tráfico de las redes sociales y 19,24\% de tráfico orgánico; Chirije $89,34 \%$ de tráfico orgánico y $10,66 \%$ de tráfico en redes sociales; las demás empresas de hospedaje se mantienen con un $100 \%$ en el tráfico orgánico.

2. El total de visitas más alto lo tiene Chirije con una representación del 77\% que corresponde a 2.400 visitas; seguido por Costas del Sol Marco's que representa un 51\% lo que corresponde a 747 visitas y Palma Azul con un 23\% que representa 721 total de visitas.

3. De las empresas de servicio de hospedaje Palma Azul representa 00.05:35 en tiempo promedio de visitas, un promedio de páginas vistas 
de $1.48 \%$, una tasa de rebote de $49,29 \%$, la participación del tráfico es por medio de un computador lo que corresponde a un $88,19 \%$ y desde un dispositivo móvil que es igual a 11,81\%; la cabaña Chirije representa 00:04:37 segundos en tiempo promedio de visitas, un promedio de páginas vistas de $1.48 \%$, una tasa de rebote de $49,29 \%$, en su mayoría el tráfico proviene desde conexiones realizadas desde un computador que representa un $75,65 \%$ y desde un dispositivo móvil un $24,35 \%$.

4. El presente análisis, da a conocer según los datos obtenidos por las dos plataformas online, que la pensión Palma Azul, la cabaña Chirije y la pensión Casa del Sol, tienen compatibilidad con dispositivos móviles.

\section{Conclusiones}

Las empresas que poseen presencia web en los buscadores solo tienen una representación de 12 empresas de servicios hoteleros que representa un $23 \%$ del total de empresas. La auditoría SEO On Page, declaró que las empresas que brindan servicio hotelero no poseen compatibilidad con dispositivos móviles, según existe una alta tendencia con el uso de dispositivos móviles con referencia al uso de computadores.

Se determinó que la pensión Palma Azul posee el mejor posicionamiento seguida por la cabaña Chirije y finalmente la pensión Casa del sol, determinando que estas empresas que brindan servicio hotelero tienen una visibilidad media en su blog corporativo, su posicionamiento podría mejorar cumpliendo con cada uno de los parámetros con fallas existentes. Las estrategias de marketing utilizadas hasta el momento no son suficientes por lo que se debe considerar la implementación del uso de marketing móvil, la utilización del mailing y la aplicación de herramientas para medir la optimización en función a consultas del usuario o estrategias de palabras claves, utilización de enlaces externos; aprovechando las oportunidades que brinda este tipo de medios. 


\section{Referencias}

Andrade, D. (2016). Estrategias de marketing digital en la promoción de marca ciudad. Revista Escuela de Administración de Negocios, (80), 59-72, e-ISSN: 0120-8160. Recuperado de:

https://doi.org/10.21158/01208160.n80.2016.1457

Cárdenas, H. (2015). Marketing Digital Una Estrategia Generadora de Innovación Empresarial Big Data Center Barcelona. Bogotá, Colombia: Universidad Santo Tomas. Recuperado de:

https://repository.usta.edu.co/bitstream/handle/11634/2815/Vargasharv ey2015.pdf? sequence $=1$ \&isAllowed $=y$

Marcos, M., \& Codina, LI. (2005). Posicionamiento web: conceptos y herramientas. El profesional de la información, 14(2), 84-99, e-ISSN: 1386-6710. Recuperado de:

https://dialnet.unirioja.es/servlet/articulo?codigo $=1121796$

MINTUR (2018). Portal de Servicios MINTUR. Ecuador: Ministerio de Turismo del Ecuador. Recuperado de:

https://servicios.turismo.gob.ec/index.php/turismo-cifras

Miranda, A., Cruz, I., Valle, M., \& Flores, J. (2015). Estrategias de marketing con redes sociales en hoteles de cuatro y cinco estrellas en la ciudad de Tijuana, Baja California. Teoría y Praxis, 10-31, e-ISSN: 1870-1582. Recuperado de:

https://www.redalyc.org/articulo.oa?id=456144904002 


\section{Frank Ángel Lemoine Quintero}

e-mail: flemoine1964@gmail.com

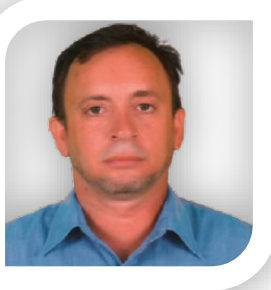

Nacido en Baracoa, Cuba, el 17 de mayo del año 1964. Docente titular de la Universidad Laica Eloy Alfaro de Manabí, Extensión Bahía de Caráquez (ULEAM), en Ecuador; Master en Administración de Empresas; Investigador acreditado por la SENESCYT REG-INV-16-01382; Responsable de la comisión de tutorías del Campus Bahía de Caráquez, Manabí; Autor de libros como Plan de Marketing, para empresas comerciales y del cliente al proveedor en Ecuador. 


\section{Cristian Eduardo Medranda Vera}

e-mail: cristian.medranda@hotmail.com

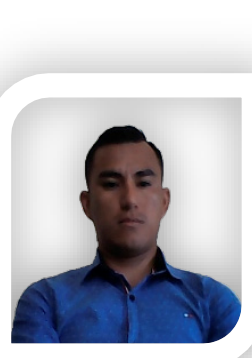

Nacido en el Cantón San Vicente, Ecuador, el 29 de mayo del año 1990. Estudiante de la Carrera de Marketing de la Extensión Bahía de Caráquez de la Universidad Laica "Eloy Alfaro" de Manabí (ULEAM), en Ecuador; Miembro del grupo científico estudiantil de la Extensión, líder del proyecto investigativo estudiantil y autor de estudios relacionados al Marketing Digital; He colaborado en proyectos institucionales destacando mi actitud de emprendimiento y he participado en eventos científicos estudiantiles a nivel nacional. 


\section{Gema Viviana Carvajal Zambrano}

e-mail: vivicarvajalzambrano@gmail.com

Nacida en San Antonio, Ecuador, el 5 de junio del año

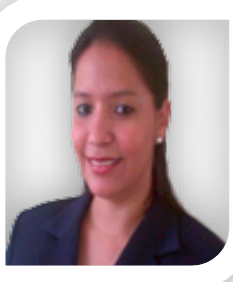
1986. Ingeniera Comercial con mención en Proyectos de Inversión; Diplomado en Marketing; Magister en Administración de Empresas con mención en Marketing (MBA); Docente en Marketing, Administración de Empresa y Hotelería de la Universidad Laica "Eloy Alfaro" de Manabí, Extensión Bahía de Caráquez (ULEAM); Destacado aporte en el desarrollo académico y de vinculación con la comunidad; Carrera de Marketing; Miembro de Proyecto de Investigación Proyecto "Observatorio Turístico para el Control de la Gestión del destino turístico Sucre, San Vicente, Jama y Pedernales" de la Extensión Bahía de Caráquez. 


\section{César Arturo Carbache Mora \\ e-mail: cesarcarbache@gmail.com}

Nacido en Guayaquil, Ecuador, el 24 de julio del año

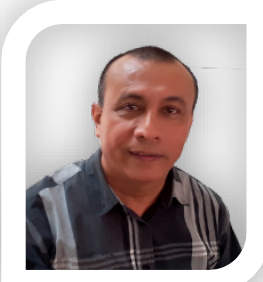
1965. Magister en Comunicación con mención en Comunicación Digital; Líder del proyecto: Aplicación del Marketing Experiencial para la Creación Promoción, Difusión y Posicionamiento post-terremoto de la nueva imagen en los destinos Sucre, San Vicente, Jama y Pedernales; Investigador Auxiliar 1 acreditado por la SENESCYT; Docente de la Universidad Laica "Eloy Alfaro" de Manabí, Extensión Bahía de Caráquez (ULEAM), Ecuador. 